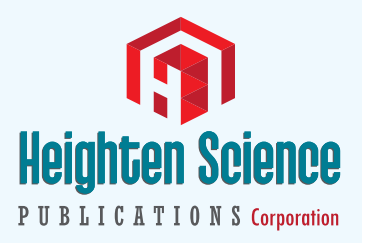

ISSN

2639-9911
*Address for Correspondence: Colin Pritchard Professor, Faculty Health \& Social Sciences, Bournemouth University UK, Tel: 07825248004; Email: cpritchard@bournemouth.ac.uk

Submitted: 13 July 2018

Approved: 16 August 2018

Published: 17 August 2018

Copyright: @ 2018 Pritchard C, et al. This is an open access article distributed under the Creative Commons Attribution License, which permits unrestricted use, distribution, and reproduction in any medium, provided the original work is properly cited

Keywords: Patients; Professional communication; Responsibility; Independence

Check for updates

\section{A Case-Study of the Anatomy of a Miscommunication: Why colleagues as patients develop complications?}

\author{
Colin Pritchard ${ }^{1 *}$ and Jeremy Latham ${ }^{2}$ \\ 'Professor, Research Professor, Faculty Health \& Social Sciences, Bournemouth University UK \\ ${ }^{2}$ Consultant, Orthopaedic Surgeon, Spire Hospital, Honorary Senior Lecturer, University of \\ Southampton, UK
}

\section{Abstract}

This is an anatomy of a miscommunication, written by the patient, a medical school professor and his orthopaedic consultant, who was also a colleague leading to a series of misunderstandings. This raises the practical question of who is responsible for effective communication with the patient who is also a colleague. At the pre-operative assessment a combination of the diffidence of an inexperienced nurse and the patient's wrong assumptions about his post-operative mobility and his keenness to maintain his independence and identity nearly led to a delayed discharge. The miscommunication was due to the patient's assumptions about previous orthopaedic and recent cardiac surgery hospital experience. Neither he nor the nurse checked these assumptions and we speculate might this possibly account for why senior colleagues who become patients sometimes have unexpected complications. There are lessons to be learned from this frank exploration of the colleague patient's experience of a miscommunication.

\section{Introduction}

The recent British Supreme Court ruling, known as the Montgomery Informed Consent case, over-ruled previous House of Lords judgements on the nature of informed consent. It was argued that what matters is not what the Doctor or Nurse thinks the patient should be told but rather, what the patient may want to know [1]. Thus, the patient becomes the centre and lead in the consent process. But implicitly, usually the patient is a 'lay' person and therefore may not be informed or have the necessary skills to ascertain what there is to be known. However, in today's world where so much information is available on the internet, the patient might well use Google, explore Medline, as well as seek further information from their nurse or physician to help them to make an informed judgement. It is recognised that at the centre of surgical complication or an adverse event across most health specialities is a breakdown in communication [1-5]. Indeed the British Chief Medical Officer in his 'Making surgery Safer' stressed such events invariably began with a break-down in communication [6] Furthermore, in the largest ever UK study of general surgeons, 549 respondents, concerned with patient safety, they stressed that most problems emerged from misunderstandings between nursing, medical or managerial colleagues [3]. However, even with near misses and minor events, in this study it was never more than $1.6 \%$ of operations [3], but if you were the patient then this represented a $100 \%$. Of the various combinations of gaps in communication between nurses, medical staff, a range of colleagues, in our search of the literature we could find no study that mentioned this particular situation of when the patient is a colleague. The present case-study is an 'anatomy of a miscommunication', authored by the patient and the consultant involved, is where the patient is a colleague. It raises the problem of when the patient is a fellow-professional and how medical and nursing staff responds to them. 
This patient is a research active Emeritus Professor of the local medical school, who it might be assumed, would have already known or crucially, be able to ask the appropriate questions. He and the consultant teach that it is not the patient's responsibility to communicate but rather it is the healthcare professional's primary role. Furthermore, we remind our students that complications and litigation are more likely to happen when communication breaks down $[7,8]$. Moreover, when the patient receives the information they may not fully understand it [9]. This makes it the duty of the healthcare professional to ensure that the patient does fully understand and though possibly time consuming it will be safer in the end. So is it different for the patient who is also a colleague? The situation arose when he became an orthopaedic patient for a second total hip replacement (THP), seventeen years after his first operation, which had been undertaken by his present colleague. The patient's research crosses many disciplines as evidenced by publications in journals such as Acta Neurological Scandinavia, British Journal of Cancer, British Journal of Neurosurgery, British Journal of Psychiatry and Child Abuse and Neglect and he is well known in his region for his friendly manner and is generally a very popular lecturer. It would be reasonable for the nurse to assume that he would have been well-informed about what was going to happen to him or would not be afraid to ask. In fact what occurred and what is described here, is an analysis of the anatomy of our miscommunication and looks at a neglected area, namely when the patient is a professional colleague. This is a frank and honest description of what went wrong, which potentially could have had serious consequences. Moreover the authors believe there are general lessons to be learned and we speculate whether our experience explains why some senior colleagues, when patients, appear to have unlikely complications?

\section{This patient's journey}

To understand the anatomy of this miscommunication, we need to explore the patient's total patient experience over time, as we need to do with all patients. He was first an in-patient in 2000 at the time of his first THR. His wife, a Principal Theatre Sister, had said "nobody likes a bolshie patient, so behave yourself". Always compliant, when asked by the nurse how he would like to be addressed, he was happy for staff to use his forename but crucially did not explain that he was a colleague from the hospital and university. Thus at 64 he was treated just like any patient. However, one-day after his operation, he had to protest to the third-year student nurse who said that she was to turn him on his un-operated side. When he questioned of how sure she was she told him she was in-charge. After some gentle discussion on how turning he on his unoperated side might damage the calcus, the nurse, realising her mistake apologised. He reassured her that this was a mistake that she would never make again and we learn from our mistakes. Though of course it could have caused serious disruption to his recovery namely damage to the healing bone. From this rather frightening experience the patient decided that if ever he was to be hospitalised again he would ensure that staff 'would know who he was', that is, he thought of himself as a colleague of the nurses and doctors. Four years later, in 2004 he was admitted as an acute emergency with an abdominal obstruction - he explained to the registrar who was consenting him for surgery, that; 'knowledge was doubled edged' and that he, as the patient, did not want to think about complications etc. "I don't want to scare myself to death so tick the boxes and I'll sign".

Furthermore, he recalled his old professor's belief that truly informed consent is a myth, as the patient can not be expected to weigh-up all the possibilities and judge the cost-benefits when in an acute, vulnerable situation and wants and needs to have trust in those caring for them [10]. We now think, this even applies even more so when the patient is a colleague! It is clearly important for the patient to be as informed as much is helpful, whoever they are - but not in an overly legalistic/managerial manner and this patient was acutely aware of the need to keep his blood pressure stable and was keen to maintain his sense of identity and control, perhaps not least to hide his anxiety. So the register complied. 
Fast forward to 2017 two months before his planned second THR was due. The patient needed a pacemaker fitted because of a degree of cardiac insufficiency and marked extra-systoles. He was extremely impressed by the fact that he was admitted to the Cardiac unit at $8 \mathrm{am}$, operated on at $11 \mathrm{am}$, was discharged and back home by $3.30 \mathrm{pm}$. 'This was cardiac surgery, albeit minor', and reflecting back over 50 clinical years, marvelled just how much cardiovascular surgery has progressed since the late 1960 's. At his assessment and preparation for his new THR, later he realised that he might have overawed the assessment nurse, although he sought to put her at her ease and they parted on seemingly very affable terms. With hind-sight however he realised that he might have talked too much as he sought to maintain control and hide his underlying anxiety. When told that he would be in hospital only 4 or 5 days, he recalled that the previous THR stay had been 10 days and when discharged, whilst he had limited mobility, he nevertheless could get in and out of bed unaided within seven days. He therefore assumed that there had been similar advances in modern orthopaedics', as with his recent cardiac experience. Hence he thought he would have a similar degree of improved recovery and mobility but after only five days. Crucially he had linked the cardiac day-case to the forthcoming orthopaedic operation and assumed a similar relative speedy recovery to independence, but he did not check out his assumption!

He is widower and lives alone and it was planned that his daughter would spend the first two days with him on his discharge. He was keen to reassure his family that after the weekend, seven days post-op, he would be able to manage. Again, neither he nor anyone of the medical or nursing staff tested out his assumptions. He had not helped himself by being keen to avoid being dependent. However, at no time did any of the professional staff ask him about what he thought would be happening and what he would be able to do unaided, or more importantly explain to him the likely degree of his immobility. Namely he would probably need help in getting in and out of bed for 2 or 3 more days after discharge, when the arrangement he had made would mean he was alone. The day before he was to be discharged he discovered his mistake and was able to make arrangements that avoided him becoming an unnecessary delayed discharge.

\section{Lessons learned}

We might ask whose 'fault' was the miscommunication? Was it the patient's - who was keen to minimise his patientstatus, eager to avoid making demands upon others and maintain his identity? But his very strengths in this situation had become a weakness. Conversely, was it because the staff were too diffident to explain simple things to the 'great personage' lest they appear to be inadvertently ageist or patronising? Senior consultants might regard it as an honour to be asked to treat equally senior colleagues, but the relationship between them is complex. It is all too easy to make assumptions about how much the patient/colleague understands and it behoves the consultant and nurse (and everyone else involved in the care pathway) to ignore the fact that the patient is a colleague. The colleague-patient is likely and understandably to be very anxious about becoming a patient, because knowledge is double-edged. They may try to cover up their fears by presenting themselves as confident in and knowledgeable about what is to be done to them. We ask, does this kind of behaviour explain why some senior colleagues when patients, experience problems because they are too proud to ask for explanations, or are not properly informed? We reiterate that it is the professional's primary responsibility clearly and fully to communicate, even if it means telling senior colleagues that they really do need to go through all aspects of their treatment. The colleague as a patient poses different kind of communication problems but we have to remember the majority of adverse events start with a miscommunication and check lists are not really enough [1-6].

\section{Conclusion}

In the final analysis we are all 'just a patient' and the colleague- patient especially, needs to have patience and a degree of acceptance that they may have things to learn. So paradoxically, we recommend that when senior professionals become patients, 
especially the more senior, then NO assumptions should be made about what they know. The nurse looking after them is the key professional and they need to go through the appropriate protocols. The nurse needs to be ready to explain what is likely to occur and assuage any possible injured pride, by pointing them towards this 'anatomy class of a miscommunication' which could have led to potential complications even for the well-informed and well-connected.

\section{Acknowledgement}

We have no conflict of interest nor was there any external funding received for this study. We are grateful for the patience and forbearance of nursing colleagues.

\section{References}

1. de Vries EN, Ramrattan MA, Smorenburg SM, Gouma DJ, Boermeester MA. The incidence and nature of in-hospital adverse events: A systematic review. Qual Saf Health Care. 2008; 17: 216-223. Ref.: https://tinyurl.com/y8pck343

2. Aoki N, Uda K, Ohta S, Kiuchi T, Fukui T. Impact of miscommunication in medical dispute cases in Japan. Int J Qual Health Care. 2008; 20: 358-362. Ref.: https://tinyurl.com/ydclelga

3. Pritchard C, Brackstone J, MacFie J. Adverse event and patient safety in the operating theatre: Perspectives of 549 surgeons. Annals of Royal College of Surgeons. 2010; 92: 1-4. Ref.: https://tinyurl.com/y79lut38

4. Weinert M, Mayer H, Zojer E. Skilled communication as "intervention": Models for systematic communication in the healthcare system. Anaesthetist. 2015; 64: 137-144. Ref.: https://tinyurl.com/y9u79vwl

5. Raman J, Leveson N, Samost AL, Dobrilovic N, Oldham M, et al. When a checklist is not enough: How to improve them and what else is needed. Journal Thoracic Cardiovascular Surgery. 2016; 152: 585592. Ref.: https://tinyurl.com/y7vv4hzv

6. Chief Medical Office. Making Surgery Safer. CMO Office. Dept of Health. 2007.

7. Chan SW, Tullocch E, Cooper ES, Smith A. Informed Consent: The Montgomery Judgement. British Medical Journal. 2017; 357: 1-3.

8. Riedl D, Schussler G. The influence of the doctor-patient communication on health outcomes: A systematic review. Psychosomatic Medicine \& Psychotherapy. 2017; 3: 131-150.

9. Halkett GK, Jiwa M, Lobb EA. Patients' perspectives on the role of the general practitioner after receiving an advanced cancer diagnosis. Eur J Cancer Care. 2015; 24: 662-672. Ref.: https://tinyurl.com/ycn4dyf7

10. Hamilton M. On Informed Consent. British Journal Psychiatry. 1983; 143: 416-418. Ref.: https://tinyurl.com/y9nrou8z 